\title{
Physical parameters of helium-rich subdwarf B stars from spectral energy distributions ${ }^{\star}$
}

\author{
A. Ahmad and C. S. Jeffery \\ Armagh Observatory, College Hill, Armagh BT61 9DG., N. Ireland, UK \\ Received 15 July 2003 / Accepted 2 October 2003

\begin{abstract}
We present effective temperatures and angular radii for eleven helium-rich subdwarf B stars. These are measured using spectral energy distributions from archival IUE spectra and existing optical and infrared photometry. The data have been analysed using a grid of high-gravity helium-rich LTE model atmospheres and a $\chi^{2}$-minimization procedure. The parameters derived here allow for independent verification of the parameters derived from the analysis of optical spectra.
\end{abstract}

Key words. stars: chemically peculiar - stars: early-type - subdwarfs - stars: atmospheres - stars: fundamental parameters

\section{Introduction}

Helium-rich subdwarf B stars (He-sdB) stars are rare faint blue stars first identified as sdOD stars in the Palomar-Green (PG) survey of faint blue objects (Green et al. 1986). They are found both in our Galaxy (PG survey) as well as in globular clusters (Moehler et al. 1997, 2002). The optical spectra of He-sdB stars, by definition show strong HeI and in some cases HeII absorption lines. Some of them also show strong carbon lines.

The evolution of these stars has been under much debate. Initially it was suggested that they might be the products of merged white dwarfs (Iben \& Tutukov 1986). More recently Brown et al. (2001) have suggested that these stars might be the product of late flash-mixing in single star evolution.

Analysis of optical spectra (Ahmad \& Jeffery 2003 Paper I) of a sample of fifteen He-sdB stars indicate that they have effective temperatures $\left(T_{\text {eff }}\right)$ in the range $30000-40000 \mathrm{~K}$, surface gravities $(\log g)$ in the range 5.0-6.0 (cgs) and helium abundances $\left(n_{\mathrm{He}}\right)$ ranging from $0.10-0.99$. Although He-sdB stars exhibit a range of helium abundance, most of them are extremely helium-rich, having $n_{\mathrm{He}} \geq 0.90$.

Misclassification of He-sdB stars is a known problem (Paper I). The PG catalogue classified cooler subdwarfs having "pure" HeI spectra with weak or absent hydrogen Balmer lines as sdOD stars implying that He-sdB stars should be extremely helium-rich. Other authors have identified sdB stars showing significantly more helium than "normal" to be He-sdB stars, e.g. JL $87\left(n_{\mathrm{He}} \sim 0.17\right.$, Schulz et al. 1991) and LS IV-14 116 $\left(n_{\mathrm{He}} \sim 0.21\right.$, Paper I). Such stars have also been analysed in this paper although they are not "typical" He-sdB stars.

This paper aims to measure the fundamental parameters effective temperature $\left(T_{\text {eff }}\right)$, interstellar reddening $\left(E_{B-V}\right)$ and

Send offprint requests to: A. Ahmad, e-mail: amir@star.arm.ac.uk

* Based on INES data from the IUE satellite. angular diameter $(\theta)$ of a sample of He-sdB stars by studying their flux distribution using a grid of helium-rich model atmospheres and a $\chi^{2}$-minimization procedure. Stars selected for this study include all He-sdB stars from the literature which have been observed with the International Ultraviolet Explorer (IUE) satellite. These observations were supplemented with optical photometric measurements from the literature and from the recently released 2 MASS infrared measurements. The parameters derived by this method are independent from those obtained from the analysis of optical spectra (Paper I), thus providing an independent verification.

\section{IUE observations}

IUE observations for eleven He-sdB stars, shown in Fig. 1, have been collected from the IUE Final Archive at MAST as "IUE Newly Extracted Spectra" (INES, Nichols \& Linsky 1996). The observations listed in Table 1 were made at low resolution $(\sim 6 \AA)$ with the Short Wavelength Prime (SWP: 1150$1980 \AA$ ) and the Long Wavelength Prime and Redundant (LWP and LWR: 1850-3350 $\AA$ ) cameras. The short wavelength cameras on the IUE were more sensitive than the long wavelength cameras and hence the SW and LW observations were merged in the overlap region (1850-1980 $\AA$ ) using weights of 100:1 with the Starlink package DIPSO (Howarth et al. 1998). For the analysis the IUE spectra were trimmed at the long and short wave limits to remove noisy data.

We found that two stars originally classified as He-sdB stars by Beers et al. (1992) and observed with the IUE are actually white dwarfs; BPS CS 29517-0049 is a DB white dwarf (Wegner \& Nelan 1987) and BPS CS 22968-0019 is a DB4 white dwarf (Wesemael et al. 1993). These two stars were not analysed. 


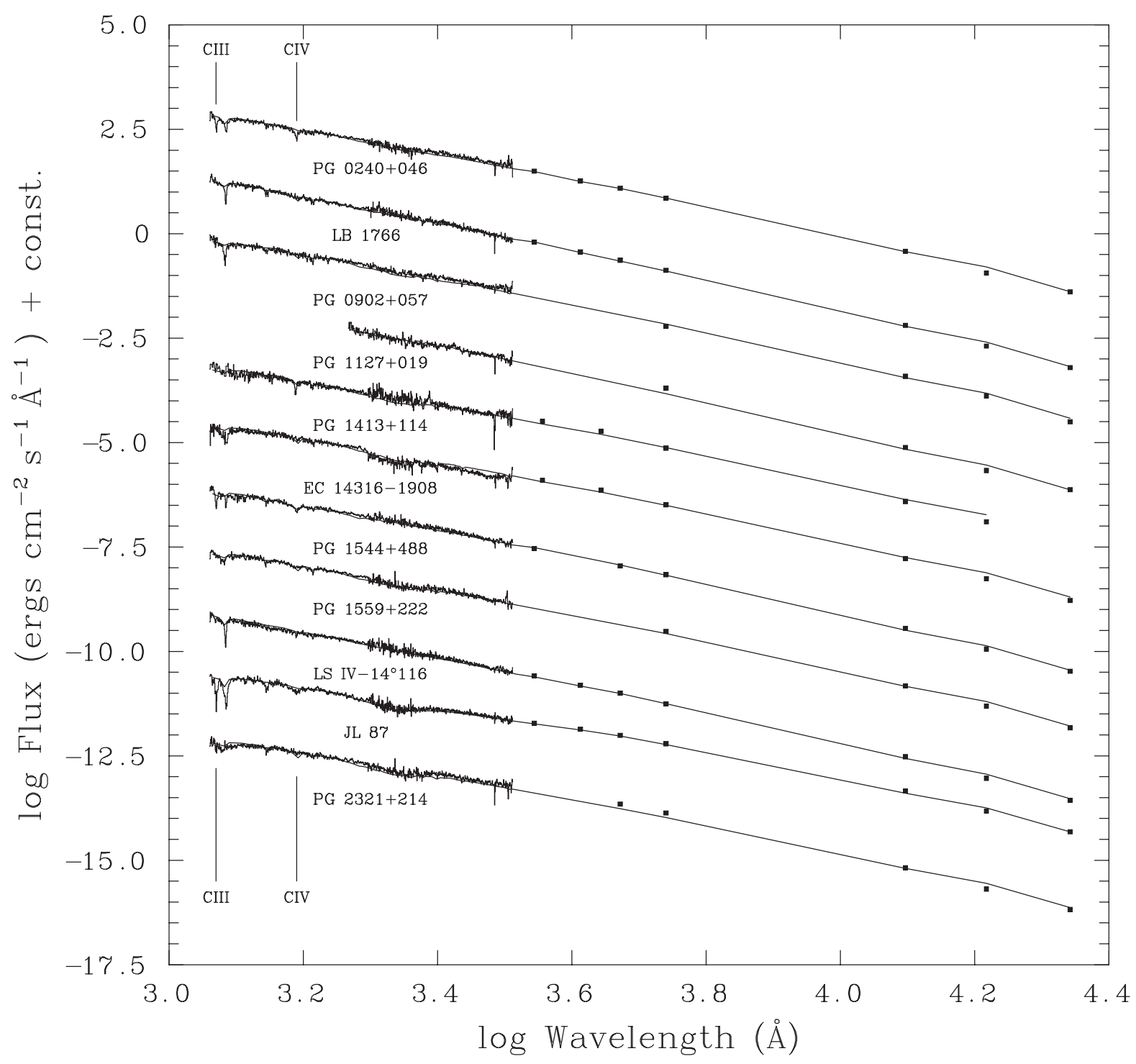

Fig. 1. Flux distribution of He-sdB stars with the model fits.

Table 1. IUE observations of He-sdB stars.

\begin{tabular}{lcccc}
\hline \hline Star & LW Image & Exp [s] & SW Image & Exp [s] \\
\hline PG 0240+046 & LWP 18452 & 2699 & SWP 39307 & 2099 \\
LB 1766 & LWP 31664 & 399 & SWP 56159 & 299 \\
PG 0902+057 & LWR 06514 & 3599 & SWP 56259 & 999 \\
& LWP 31781 & 1499 & - & - \\
PG 1127+019 & LWP 10994 & 1199 & - & - \\
PG 1413+114 & LWP 10989 & 8999 & SWP 31135 & 14999 \\
EC 14316-1908 & LWP 23384 & 1320 & SWP 45020 & 660 \\
PG 1544+488 & LWP 10992 & 899 & SWP 31142 & 899 \\
PG 1559+222 & LWP 10993 & 6899 & SWP 31143 & 5999 \\
LS IV-14¹16 & LWP 10814 & 759 & SWP 31029 & 759 \\
JL 87 & LWP 09467 & 419 & SWP 29594 & 299 \\
PG 2321+214 & LWP 18329 & 1445 & SWP 39208 & 1019 \\
\hline
\end{tabular}

\section{Photometry}

Photometric measurements of $\mathrm{He}-\mathrm{sdB}$ stars were collected from published photometry. The optical photometry includes Johnson $U B V$ and Strömgren uvby measurements and these are listed in Table 2. Infrared Johnson $J H K$ measurements, listed in Table 3 were collected from the 2MASS All-Sky Catalogue of Point Sources (Cutri et al. 2003).

For some of the stars there are no published $y$ or $V$ measurements; in such cases the $V$ magnitude has been calculated from the $B_{\text {pg }}$ magnitude using the transformation equation given by Thejll et al. (1994),

$V=6.628 \times 10^{0.024 B_{\mathrm{pg}}}$.

The transformed $V$ magnitudes have a standard deviation of $0.3 \mathrm{mag}$. The errors in the photometric measurements listed in Tables 2 and 3 are derived from the source papers.

The photometric magnitudes used in the energy distribution analysis were converted into fluxes using

$F_{\lambda}=10^{0.4\left(C_{\lambda}-m_{\lambda}\right)}$.

The scale factors $C_{\lambda}$ used for converting the magnitudes to fluxes are taken from Heber et al. (1984) for the Strömgren uvby filters and from Johnson (1966) for the Johnson UBVJHK filters. 
Table 2. Visible light photometry of of He-sdB stars.

\begin{tabular}{|c|c|c|c|c|c|c|}
\hline Star & $u$ & $v$ & $b$ & $U$ & $B$ & $y / V$ \\
\hline PG 0240+046 & $13.785 \pm 0.021^{1}$ & $14.034 \pm 0.018^{1}$ & $14.037 \pm 0.016^{1}$ & - & - & $14.142 \pm 0.014^{1}$ \\
\hline LB 1766 & $11.906 \pm 0.112^{2}$ & $12.158 \pm 0.053^{2}$ & $12.223 \pm 0.023^{2}$ & - & - & $12.343 \pm 0.012^{2}$ \\
\hline PG 0902+057 & - & - & - & - & - & $14.415 \pm 0.300^{3}$ \\
\hline PG $1127+019$ & - & - & - & - & - & $13.603 \pm 0.300^{3}$ \\
\hline PG $1413+114$ & - & - & - & $14.580 \pm 0.360^{4}$ & $15.710 \pm 0.024^{4}$ & $16.070 \pm 0.015^{4}$ \\
\hline EC 14316-1908 & - & - & - & $11.860 \pm 0.040^{5}$ & $12.990 \pm 0.020^{5}$ & $13.210 \pm 0.010^{5}$ \\
\hline PG $1544+488$ & $12.500 \pm 0.120^{6}$ & - & $12.770 \pm 0.070^{6}$ & - & - & $12.800 \pm 0.050^{6}$ \\
\hline PG $1559+222$ & - & - & - & - & - & $14.786 \pm 0.300^{3}$ \\
\hline LS IV-14 116 & $12.629 \pm 0.025^{2}$ & $12.847 \pm 0.011^{2}$ & $12.882 \pm 0.003^{2}$ & - & - & $13.029 \pm 0.001^{2}$ \\
\hline JL 87 & $12.082 \pm 0.056^{7}$ & $12.095 \pm 0.032^{7}$ & $12.034 \pm 0.026^{7}$ & - & - & $12.049 \pm 0.022^{7}$ \\
\hline PG $2321+214$ & - & - & $13.530 \pm 0.364^{8}$ & - & - & $13.560 \pm 0.324^{8}$ \\
\hline
\end{tabular}

References :

${ }^{1}$ Wesemael et al. (1992); ${ }^{2}$ Kilkenny \& Busse (1992); ${ }^{3} V$ calculated using Eq. (1); ${ }^{4}$ Beers et al. (1992); ${ }^{5}$ Kilkenny et al. (1997); ${ }^{6}$ Green (1980); ${ }^{7}$ Hauck \& Mermilliod (1998); ${ }^{8}$ Bixler et al. (1991).

Table 3. Infrared photometry of He-sdB stars from the 2MASS survey (Cutri et al. 2003).

\begin{tabular}{lccc}
\hline \hline Star & $J$ & $H$ & $K$ \\
\hline PG 0240+046 & $14.755 \pm 0.033$ & $14.936 \pm 0.052$ & $14.844 \pm 0.100$ \\
LB 1766 & $13.062 \pm 0.027$ & $13.181 \pm 0.031$ & $13.248 \pm 0.038$ \\
PG 0902+057 & $14.742 \pm 0.036$ & $14.789 \pm 0.049$ & $15.116 \pm 0.162$ \\
PG 1127+019 & $14.498 \pm 0.029$ & $14.751 \pm 0.062$ & $14.688 \pm 0.111$ \\
PG 1413+114 & $16.609 \pm 0.138$ & $16.701 \pm 0.316$ & - \\
EC 14316-1908 & $13.773 \pm 0.026$ & $13.848 \pm 0.045$ & $13.933 \pm 0.060$ \\
PG 1544+488 & $13.460 \pm 0.022$ & $13.561 \pm 0.030$ & $13.666 \pm 0.046$ \\
PG 1559+222 & $15.398 \pm 0.054$ & $15.482 \pm 0.112$ & $15.555 \pm 0.219$ \\
LS IV-14 116 & $13.638 \pm 0.027$ & $13.799 \pm 0.031$ & $13.898 \pm 0.051$ \\
JL 87 & $12.308 \pm 0.026$ & $12.388 \pm 0.027$ & $12.410 \pm 0.026$ \\
PG 2321+214 & $14.289 \pm 0.024$ & $14.430 \pm 0.038$ & $14.434 \pm 0.075$ \\
\hline
\end{tabular}

\section{Models and $\chi^{2}$ minimization}

A grid of LTE model atmospheres was computed assuming plane-parallel geometry and hydrostatic equilibrium using the code STERNE (Jeffery \& Heber 1992). The grid points were defined by $T_{\text {eff }}=20000(5000) 40000,50000, \log g=$ $5.0(0.5) 6.0$ and $n_{\mathrm{He}}=0.100,0.300,0.699,0.997$. Solar metal abundances were assumed. Another model grid was used to study the effect of carbon enhancement in the model atmospheres, these comprised $T_{\text {eff }}=28000(2000) 40000$, $\log g=5.0(0.5) 6.0$ and abundance $\left(n_{\mathrm{He}}+n_{\mathrm{C}}\right)=0.960+0.030$, $0.990+0.010,1.000+0.003$.

The FORTRAN90 program FFIT (Jeffery et al. 2001) was used to calculate the effective temperature $\left(T_{\text {eff }}\right)$, reddening $\left(E_{B-V}\right)$ and angular diameter $(\theta)$ from the observed flux distribution by fitting model fluxes using a $\chi^{2}$ minimisation procedure (cf. Aznar Cuadrado \& Jeffery 2001).
The best fit parameters for each star correspond to the minimum in the $\chi^{2}$ surface. Aznar Cuadrado \& Jeffery (2001) had noted that these surfaces should be treated carefully as they may have more than one minimum and FFIT can find wrong parameters if it ends up in a local minimum instead of the global minimum. We examined the quality of each final solution (Fig. 1) to ensure that it looked reasonable and to check whether it was consistent with previous measurements.

\section{Errors}

Aznar Cuadrado \& Jeffery (2001) have shown that the $T_{\text {eff }}$ is not very sensitive to $\log g$ in the range of 5.0-6.0 (cgs). We have therefore assumed a value for $\log g$ for our stars based on Paper I and previous analyses. The results do not change if $\log g$ is varied by \pm 0.3 dex. Also, $n_{\mathrm{He}}$ does not affect the parameters if it is varied by \pm 0.1 . The best model fits were calculated 
Table 4. Physical parameters of of He-sdB stars along with previous measurements from the literature. Values in square brackets are assumed.

\begin{tabular}{|c|c|c|c|c|c|c|}
\hline Star & $\bar{E} E_{B-V}$ & $\begin{array}{l}\theta \text { [ [rad] } \\
\times 10^{-12}\end{array}$ & $\bar{T} T_{\text {eff }}[\mathrm{K}]$ & $\log g$ [cgs] & $n_{\mathrm{He}}$ & Reference \\
\hline PG 0240+046 & $0.07 \pm 0.01$ & $4.08 \pm 0.09$ & $34600 \pm 1700$ & {$[5.5]$} & {$[0.70]$} & FFIT \\
\hline \multirow[t]{4}{*}{ [carbon-rich] } & - & - & $34000 \pm 150$ & $5.40 \pm 0.10$ & $0.63 \pm 0.01$ & Paper I \\
\hline & - & - & $36200 \pm 400$ & $6.25 \pm 0.10$ & $0.66 \pm 0.02$ & Aznar Cuadrado \& Jeffery (2002) \\
\hline & 0.06 & - & $34800 \pm 1850$ & - & - & Aznar Cuadrado \& Jeffery (2001) \\
\hline & - & - & $37000 \pm 2000$ & $5.30 \pm 0.30$ & $0.55 \pm 0.11$ & Thejll et al. (1994) \\
\hline LB 1766 & $0.02 \pm 0.01$ & $7.98 \pm 0.17$ & $40400 \pm 1300$ & {$[6.0]$} & {$[0.99]$} & FFIT \\
\hline [carbon-poor] & - & - & $40000 \pm 2000$ & $6.30 \pm 0.30$ & 0.99 & Lanz et al. (2003) \\
\hline PG 0902+057 & $0.08 \pm 0.01$ & $3.37 \pm 0.10$ & $47000 \pm 3000$ & {$[6.0]$} & [0.99] & FFIT \\
\hline \multirow{2}{*}{ [carbon-poor] } & - & - & $>40000$ & - & - & Paper I \\
\hline & - & - & $44000 \pm 2000$ & $6.00 \pm 0.10$ & $0.97 \pm 0.19$ & Thejll et al. (1994) \\
\hline PG $1127+019$ & $0.03 \pm 0.01$ & $3.82 \pm 0.16$ & $42100 \pm 2000$ & {$[5.0]$} & {$[0.99]$} & FFIT \\
\hline [carbon-rich] & - & - & $39900 \pm 200$ & $5.00 \pm 0.10$ & $0.99 \pm 0.01$ & Paper I \\
\hline PG $1413+114$ & $0.12 \pm 0.02$ & $1.74 \pm 0.07$ & $40300 \pm 2500$ & {$[5.5]$} & {$[0.99]$} & FFIT \\
\hline [carbon-rich] & 0.00 & - & 31600 & - & - & Beers et al. (1992) \\
\hline EC 14316-1908 & $0.12 \pm 0.02$ & $6.06 \pm 0.22$ & $42000 \pm 3000$ & {$[5.5]$} & {$[0.99]$} & FFIT \\
\hline \multirow[t]{2}{*}{ [carbon-poor] } & 0.12 & - & 77000 & - & - & Drilling \& Beers (1995) \\
\hline & 0.08 & - & 33900 & - & - & Beers et al. (1992) \\
\hline PG 1544+488 & $0.01 \pm 0.01$ & $7.51 \pm 0.12$ & $32100 \pm 1000$ & {$[5.0]$} & {$[0.99]$} & FFIT \\
\hline \multirow{3}{*}{ [carbon-rich] } & - & - & $36000 \pm 2000$ & $6.00 \pm 0.30$ & 0.98 & Lanz et al. (2003) \\
\hline & - & - & $34000 \pm 300$ & $5.10 \pm 0.10$ & $0.99 \pm 0.01$ & Paper I \\
\hline & - & - & 31000 & 5.10 & $\leq 0.99$ & Heber et al. (1988) \\
\hline PG $1559+222$ & $0.09 \pm 0.02$ & $2.92 \pm 0.13$ & $38700 \pm 2100$ & {$[5.5]$} & {$[0.99]$} & FFIT \\
\hline [carbon-poor] & - & - & - & - & - & - \\
\hline LS IV-14ำ 116 & $0.03 \pm 0.01$ & $6.90 \pm 0.10$ & $32500 \pm 700$ & [5.5] & {$[0.30]$} & FFIT \\
\hline \multirow[t]{3}{*}{ [carbon-poor] } & - & - & $32500 \pm 150$ & $5.40 \pm 0.10$ & $0.21 \pm 0.01$ & Paper I \\
\hline & - & - & 35000 & - & - & Ulla \& Thejll (1998) \\
\hline & 0.01 & - & $33000 \pm 1000$ & $5.80 \pm 0.20$ & $0.20 \pm 0.07$ & Viton et al. (1991) \\
\hline JL 87 & $0.16 \pm 0.01$ & $15.00 \pm 0.36$ & $28100 \pm 1100$ & {$[5.0]$} & {$[0.10]$} & FFIT \\
\hline \multirow[t]{3}{*}{ [carbon-rich] } & - & - & $29000 \pm 2000$ & $5.50 \pm 0.30$ & $0.09-0.16$ & Lanz et al. (2003) \\
\hline & - & - & 30000 & 5.00 & - & Magee et al. (1998) \\
\hline & 0.15 & - & $28000 \pm 1000$ & $5.20 \pm 0.30$ & $0.17 \pm 0.05$ & Schulz et al. (1991) \\
\hline PG 2321+214 & $0.13 \pm 0.02$ & $4.93 \pm 0.22$ & $38400 \pm 3000$ & {$[5.5]$} & {$[0.99]$} & FFIT \\
\hline \multirow[t]{2}{*}{ [carbon-poor] } & - & - & $39600 \pm 150$ & $5.30 \pm 0.10$ & $0.99 \pm 0.01$ & Paper I \\
\hline & $0.10 \pm 0.03$ & - & $43000^{1}$ & - & - & Ulla \& Thejll (1998) \\
\hline
\end{tabular}

${ }^{1}$ Thejll, Husfeld \& Saffer (unpublished) reported by Ulla \& Thejll (1998).

without any interpolation in $\log g$ and $n_{\mathrm{He}}$. For some stars in this study there are no previous analyses for surface gravity and helium abundance, in such cases we have assumed $\log g=$ 5.5 and $n_{\mathrm{He}}=0.99$, which are the typical values for He-sdB stars (Paper I).

It is clear from optical and IUE spectra that some He-sdB stars are carbon-rich while others are carbon-poor. Hence we studied the effect of carbon enhancement in the atmosphere by adding $3 \%, 1 \%$ and $0.3 \%$ carbon in the model atmospheres. The results indicate that increasing the amount of carbon in the atmosphere does not significantly change the flux distribution in the wavelengths under study (1180-22000 $\AA$ ). The effect of the change in carbon abundance on $T_{\text {eff }}$ is $\leq 600 \mathrm{~K}$, which is quite small considering the errors associated with $T_{\text {eff }}$ in the best model fit are around $\pm 1500 \mathrm{~K}$. Hence, we have used a solar carbon abundance for this analysis.

It has been established (Kudritzki 1979) that for $T_{\text {eff }} \leq$ $35000 \mathrm{~K}$, departure from local thermodynamic equilibrium (LTE) does not significantly affect measurements of $T_{\text {eff }}$ in high-gravity stars. For hotter stars, NLTE effects become increasingly important. However the relative temperature differences obtained from LTE analysis are still useful.

\section{Results}

The IUE spectra of He-sdB stars are shown in Fig. 1 combined with the photometric fluxes and model fits. The best fit parameters derived from FFIT are quoted in Table 4 along with the formal errors.

It is interesting to note that some He-sdB stars show strong CIII/CIV lines in their IUE spectrum (see Fig. 1) as well as in the optical spectrum (Paper I) indicating that they are carbonrich. In other stars these strong carbon lines are absent. This reinforces the view that there are two distinct sub-classes within He-sdB stars, one which consists of stars which are carbonrich and the other which comprises stars which are carbon-poor (Table 4). 


\section{Discussion}

Some of our He-sdB stars have been analysed previously. For these, most of our results match quite well with those already in the literature (Table 4). PG 1413+114, EC 14316-1908 and PG $1559+222$ do not have previous estimates for $\log g$ and $n_{\mathrm{He}}$ hence typical values for these parameters were assumed for the analysis. The optical spectra of these three stars show strong HeII lines indicating that they are hot $(\gtrsim 40000 \mathrm{~K})$ and enriched in helium.

PG $1413+114$ is a He-sdB star from Jeffery et al. (1996). Beers et al. (1992) have also referred to it as a He-sdB star (BPS CS 22883-0015) and estimated $T_{\text {eff }}=31600 \mathrm{~K}$ and $E_{B-V}$ $=0.0$. Such a low effective temperature is not consistent with the presence of the strong HeII lines. We derive a much higher effective temperature and interstellar reddening for this star in our analysis.

EC 14316-1908 is a He-sdB star from the Edinburgh-Cape survey of blue objects by Kilkenny et al. (1997). Beers et al. (1992) refer to it as a He-sdO star (BPS CS 22871-0019) and estimate $T_{\text {eff }}=33900 \mathrm{~K}$ and $E_{B-V}=0.08$. This star appears as two different object in SIMBAD. Drilling \& Beers (1995) have estimated $T_{\text {eff }}=77000 \mathrm{~K}$ and $E_{B-V}=0.12$ by analysing the flux distribution of this star. The optical spectrum shows strong HeII but also some HeI which suggests that although this star is quite hot $\left(T_{\text {eff }} \geq 40000 \mathrm{~K}\right)$, it is not as hot as estimated by Drilling \& Beers (1995). We derive the same interstellar reddening but a much lower effective temperature $(42000 \mathrm{~K})$.

\section{Conclusion}

We have analysed a sample of eleven He-sdB stars and derived fundamental parameters from their flux distributions. The results confirm those obtained from analyses of optical spectra.

The IUE spectra of He-sdB stars reinforces the idea of two distinct subclasses of these stars. The carbon-rich He-sdB stars which show strong carbon lines in their optical and IUE spectra can be explained by the merger of $\mathrm{CO}+\mathrm{He}$ white dwarfs (Saio \& Jeffery 2002) as well as the flash-mixing model (Brown et al. 2001) whereas carbon-poor He-sdB stars can be explained by $\mathrm{He}+\mathrm{He}$ white dwarf mergers (Saio \& Jeffery 2000). Detailed abundance measurements will be required to determine which of these evolutionary channel(s) are responsible for the formation of these rare faint blue stars.

Acknowledgements. We thank Dr D. Kilkenny, the referee, for useful comments and suggestions. This research is supported by a grant to the Armagh Observatory from the Northern Ireland Department of Culture, Arts and Leisure. The authors acknowledge the data analysis facilities provided by the Starlink Project which is run by CCLRC on behalf of PPARC. This research has made use of NASA's Astrophysics Data System. This research has also made use of the Aladin, Vizier and SIMBAD databases, operated at CDS, Strasbourg, France. This publication makes use of data products from the Two Micron All Sky Survey, which is a joint project of the University of Massachusetts and the Infrared Processing and Analysis Center/California Institute of Technology, funded by the National Aeronautics and Space Administration and the National Science Foundation. Some of the data presented in this paper were obtained from the Multimission Archive at the Space Telescope Science Institute (MAST). STScI is operated by the Association of Universities for Research in Astronomy, Inc., under NASA contract NAS5-26555. Support for MAST for non-HST data is provided by the NASA Office of Space Science via grant NAG5-7584 and by other grants and contracts.

\section{References}

Ahmad, A., \& Jeffery, C. S. 2003, A\&A, 402, 335

Aznar Cuadrado, R., \& Jeffery, C. S. 2001, A\&A, 368, 994

Aznar Cuadrado, R., \& Jeffery, C. S. 2002, A\&A, 385, 131

Beers, T. C., Doinidis, S. P., Griffin, K. E., Preston, G. W., \& Shectman, S. A. 1992, AJ, 103, 267

Bixler, J. V., Bowyer, S., \& Laget, M. 1991, A\&A, 250, 370

Brown, T. M., Sweigart, A. V., Lanz, T., Landsman, W. B., \& Hubeny, I. 2001, ApJ, 562, 368

Cutri, R. M., Skrutskie, M. F., van Dyk, S., et al. 2003, The 2MASS All-Sky Catalogue of Point Sources

Drilling, J. S., \& Beers, T. C. 1995, ApJ, 446, L27

Green, R. F. 1980, ApJ, 238, 685

Green, R. F., Schmidt, M., \& Liebert, J. 1986, ApJS, 61, 304

Hauck, B., \& Mermilliod, M. 1998, A\&AS, 129, 431

Howarth, I. D., Murray, J., Mills, D., \& Berry, D. S. 1998, Starlink User Note, 50

Heber, U., Hunger, K., Jonas, G., \& Kudritzki, R. P. 1984, A\&A, 130, 119

Heber, U., Dreizler, S., de Boer, K. S., Moehler, S., \& Richtler, T. 1988, Astron. Gesellschaft Abstract Ser., 1, 16

Iben, I. J., \& Tutukov, A. V. 1986, ApJ, 311, 753

Jeffery, C. S., Heber, U. 1992, A\&A, 260, 133

Jeffery, C. S., Heber, U., Hill, P. W., et al. 1996, in Hydrogen Deficient Stars, ed. C. S. Jeffery, \& U. Heber, ASP Conf. Ser., 96, 471

Jeffery, C. S., Woolf, V. M., \& Pollacco, D. L. 2001, A\&A, 376, 497

Johnson, H. L. 1966, ARA\&A, 4, 193

Kilkenny, D., \& Busse, J. 1992, MNRAS, 258, 57

Kilkenny, D., O’Donoghue, D., Koen, C., Stobie, R. S., \& Chen, A. 1997, MNRAS, 287, 867

Kudritzki, R. P. 1979, The elements and their isotopes in the universe, 22nd Liège International Symp., 295

Lanz, T., Brown, T. M., Sweigart, A. V., Hubeny, I., \& Landsman, W. B. 2003, ApJ, in press

Magee, H. R. M., Dufton, P. L., Keenan, F. P., et al. 1998, A\&A, 338, 85

Moehler, S., Heber, U., \& Durrell, P. R. 1997, A\&A, 317, L83

Moehler, S., Sweigart, A. V., Landsman, W. B., \& Dreizler, S. 2002, A\&A, 395, 37

Nichols, J. S., \& Linsky, J. L. 1996, AJ, 111, 517

Saio, H., \& Jeffery, C. S. 2000, MNRAS, 313, 671

Saio, H., \& Jeffery, C. S. 2002, MNRAS, 333, 121

Schulz, H., Heber, U., \& Wegner G. 1991, PASP, 103, 435

Thejll, P., Bauer, F., Saffer, R., et al. 1994, ApJ, 433, 819

Ulla, A., \& Thejll, P. 1998, A\&AS, 132, 1

Viton, M., Deleuil, M., Tobin, W., Prévot, L., \& Bouchet, P. 1991, A\&A, 242, 175

Wegner, G., \& Nelan, E. P. 1987, ApJ, 319, 916

Wesemael, F., Fontaine, G., Bergeron, P., Lamontagne, R., \& Green, F. F. 1992, AJ, 104, 203

Wesemael, F., Greenstein, J. L., Liebert, J., et al. 1993, PASP, 105, 761 